\title{
CRISPR whole-genome screening identifies new necroptosis regulators and RIPK1 alternative splicing
}

\author{
Marinella G. Callow ${ }^{1}$, Colin Watanabe², Katherine E. Wickliffe ${ }^{3}$, Russell Bainer², Sarah Kummerfield², Julie Weng ${ }^{1}$, \\ Trinna Cuellar, ${ }^{4,6}$, Vasantharajan Janakiraman ${ }^{4}$, Honglin Chen ${ }^{4}$, Ben Chih ${ }^{5}$, Yuxin Liang ${ }^{4}$, Benjamin Haley ${ }^{4}$, \\ Kim Newton ${ }^{3}$ and Michael R. Costa ${ }^{1}$
}

\begin{abstract}
The necroptotic cell death pathway is a key component of human pathogen defense that can become aberrantly derepressed during tissue homeostasis to contribute to multiple types of tissue damage and disease. While formation of the necrosome kinase signaling complex containing RIPK1, RIPK3, and MLKL has been extensively characterized, additional mechanisms of its regulation and effector functions likely remain to be discovered. We screened 19,883 mouse protein-coding genes by CRISPR/Cas9-mediated gene knockout for resistance to cytokine-induced necroptosis and identified 112 regulators and mediators of necroptosis, including 59 new candidate pathway components with minimal or no effect on cell growth in the absence of necroptosis induction. Among these, we further characterized the function of PTBP1, an RNA binding protein whose activity is required to maintain RIPK1 protein abundance by regulating alternative splice-site selection.
\end{abstract}

\section{Introduction}

Multiple pathways in multicellular organisms have evolved to induce and execute cell death in defective, infected, or extraneous cells, and dysregulation of numerous steps in each pathway contribute to diseases and tissue damage ${ }^{1,2}$. Necroptosis is a caspaseindependent form of programmed necrosis that mediates defense against viral pathogen infection, particularly when caspase activation is blocked, by directly lysing infected cells and releasing damage-associated molecular patterns (DAMPs) to initiate an inflammatory response ${ }^{3,4}$. While clinical biomarkers of necroptosis have not yet been widely employed in human studies, necroptosis is

Correspondence: Michael R. Costa (mcosta@gene.com)

'Department of Discovery Oncology, Genentech, Inc., 1 DNA Way, South San Francisco, CA 94080, USA

2Department of Bioinformatics and Computational Biology, Genentech, Inc., 1 DNA Way, South San Francisco, CA 94080, USA

Full list of author information is available at the end of the article Edited by $\mathrm{G}$. Raschellà pathologically activated in certain mouse models of a variety of diseases and disorders involving tissue injury, degeneration, and inflammation ${ }^{5,6,7}$. Notably, genetic or pharmacologic inhibition of the necroptosis mediators RIPK1 kinase activity, RIPK3, or MLKL demonstrates therapeutic benefit in mouse models of inflammatory bowel disease, ischemia-reperfusion injury, drug-induced liver injury, acute kidney injury, retinal detachment, chronic dermatitis, atherosclerosis, and amyotrophic lateral sclerosis (ALS) ${ }^{6,7}$. However, RIPK1 enzymatic activity and Ripk 3 can also mediate apoptosis in certain contexts, and $M l k l$ deficiency is less protective than Ripk3 deficiency or catalytically inactive RIPK1 in some tissue injury and inflammation models.

Necroptosis is induced when caspase- 8 is inhibited and either a TNF family death-domain receptor, Toll-like receptor TLR3 or TLR4, or the virus sensing adaptor protein ZBP1 is triggered. RIPK1 (recruited to death receptors), TRIF (recruited to TLR3 and TLR4), and ZBP1 
are RHIM domain-containing proteins that activate the kinase RIPK3 through homotypic interaction with its RHIM domain ${ }^{9,10}$. Autophosphorylated RIPK3 in turn phosphorylates the pseudokinase MLKL to induce a conformational change, homo-oligomerization, and translocation to the plasma membrane to promote membrane permeabilization and cell lysis ${ }^{11-13}$. However, necroptosis is actively suppressed during development and in many adult tissues, such that in most cell types TNFR1 activation does not result in cell death. In a context-dependent manner, RIPK1 function is regulated at multiple checkpoints by association of RIPK1 with different binding partners in alternative complexes ${ }^{9,14,15}$. In the most completely characterized example of pathway activation, engagement of TNFR1 by the cytokine TNF $\alpha$ assembles complex-I at the cell membrane by recruiting RIPK1, TRADD, TRAF2/5, and the E3 ubiquitin ligases cIAP1/2 and LUBAC that ubiquitylate RIPK1 and other components of complex- $\mathrm{I}^{9,14-16}$. In a kinase-independent manner, ubiquitylated RIPK1 recruits and activates IKK and TAK1-TAB2/3 complexes, stimulating NF- $\mathrm{kB}$, JNK, p38, and ERK pathways to transcriptionally activate antiapoptotic and pro-inflammatory genes, including CFLAR (cFLIP) encoding the catalytically inactive homolog of caspase-8. Under conditions where RIPK1 is not efficiently ubiquitylated, complex-I is unstable, and RIPK1 dissociates to form cytoplasmic complex-II with FADD and caspase- 8 in a RIPK1 kinase-dependent manner. RIPK1 ubiquitylation can be restricted by cIAP inhibition or by the deubiquitylase activity of CYLD, which is recruited to the complex-I through the LUBAC-binding protein SPATA2 ${ }^{17-22}$. Activated caspase-8 within complex-II triggers apoptosis by cleaving executioner caspases. However, if caspase-8 expression is limited or its activity is blocked by pan-caspase inhibitors such as zVAD-fmk, then complex-II can further develop to form the necrosome complex as RIPK1 recruits and activates RIPK3, leading to MLKL-dependent necroptosis ${ }^{3,9,10,15}$.

Despite current knowledge of the RIPK1 and necroptosis signaling pathways, many aspects of their regulatory mechanisms remain to be elucidated, including ubiquitin editing dynamics, protein complex intracellular trafficking, and membrane channel interactions. Expression levels of RIPK1, RIPK3, and MLKL correlate with sensitivity to necrosis in some mouse models and human disease tissues ${ }^{2}$, and defining their transcriptional and post-transcriptional control could help verify a primary causal relationship. To discover new pathway components that might ultimately reveal new therapeutic intervention strategies or predictive biomarkers for inhibitors in preclinical development, we performed a whole-genome CRISPR screen selecting for necroptosis resistance. This screen successfully identified 59 new candidate regulatory genes previously implicated in diverse cellular functions that, similar to RIPK1, RIPK3, and MLKL, display minimal or no requirement for normal cell proliferation. We further defined the screen hit Ptbp1 as a determinant of RIPK1 protein expression levels by demonstrating that it represses alternative splicing to a cryptic exon that induces a frame shift in the Ripk1 transcript.

\section{Results}

Genome-wide CRISPR screening identifies new necroptosis pathway regulators

We developed a screen in L929 mouse fibroblast cells to discover new genes whose products mediate or modulate the induction and execution of necroptosis stimulated by recombinant TNF $\alpha$ protein in the presence of the pancaspase inhibitor zVAD-fmk ${ }^{23}$. To do so, we generated a transgenic cell line stably expressing Cas9 and validated that co-expression of a sgRNA targeting $M l k l$ reduced MLKL protein to undetectable levels and conferred nearly complete resistance to necroptosis within 8 days (Figure S1). We used this cell line to screen a novel whole-genome sgRNA library evenly partitioned in nine pools targeting an average of 2203 genes, with 93\% of genes targeted by eight sgRNAs (Figure S2). sgRNA sequences were selected using an algorithm that designs sgRNAs and prioritizes them at the gene level on the basis of the number of splice isoforms targeted, proximity to the $5^{\prime}$ end of the coding region, and the number of predicted off-target sites (see Materials and methods). Three of the library pools were screened individually at 1000 -fold sgRNA representation with a target multiplicity of infection (MOI) of 0.3-0.6. The remaining six libraries were screened as three combinations of two pools at 500-fold sgRNA representation and MOI 0.6, after determining that these parameters did not significantly reduce the screen hit detection power (Table S7). Each library contained at least one sgRNA targeting $M l k l$ as a positive control, as well as containing a common set of 47 non-targeting control (NTC) sgRNAs. For each of the six screens, three replicate samples were transduced and independently maintained at the indicated sgRNA representation through three passages over 10 days to allow for gene knockout. A cell number that maintained 500-1000-fold sgRNA representation was collected for a reference time point 2 days after transduction, and puromycin was added to an equivalent number of cells to select for sgRNA integrations starting 3 days after transduction. Ten days after transduction, $\sim 1.5 \times 10^{8}$ cells per replicate were treated with TNF $\alpha$ and zVAD-fmk to achieve $99.0-99.9 \%$ cell killing by necroptosis, and resistant cells were harvested 5 days later. In parallel, untreated cultures were maintained at the indicated sgRNA representation through an additional passage and collected at the same time as the treated samples. 
Genomic DNA (gDNA) extracted from the reference, treated, and untreated cell collections was used in an amount that maintained the sgRNA representation for PCR amplification of the sgRNA sequences. Read counts for the individual sgRNAs were determined by nextgeneration sequencing (NGS) of the PCR amplicons, after correcting for library sequencing depth and scaling the median sgRNA read count number across libraries (Table S1; see Materials and methods). The very strong level of positive selection achieved in the screens with $\mathrm{TNF} \alpha$ and zVAD-fmk provides a large assay window of over 1000-fold sgRNA enrichment, although it also produces a relatively high level of sgRNA read count variation between some replicates of only the treated samples, presumably due to stochastic differences in survival of small numbers of cells with sgRNAs that do not confer resistance (Fig. 1 and S3). The gCrisprTools software ${ }^{24}$ was used to summarize sgRNA-level signals into genelevel estimates (Rho scores) of the significance of sgRNA enrichment in the treated samples compared to the timematched untreated controls, or of sgRNA depletion in the untreated controls compared to the early reference time point samples. This analysis effectively prioritizes genes targeted by sgRNAs that are consistently enriched across replicate treated samples relative to their paired untreated controls. A total of 112 genes in the genome-wide screens show Rho scores less than $10^{-9}$ and are considered further here (Table S2 and Figure S4a).

Among the samples treated with TNF $\alpha$ and zVAD-fmk, we observe the highest level of sgRNA enrichment (up to 1260-fold) among sgRNAs targeting the gene that encodes TNF receptor Tnfrsfla (Tnfr1) and the three canonical components of the necrosome, Ripk1, Ripk3, and $M l k l$ (Fig. 2). Almost all sgRNAs targeting the genes encoding the deubiquitylase CYLD and its recently identified binding partner, SPATA2, are also strongly enrichment, consistent with the reported roles of CYLD and SPATA2 in deubiquitylating RIPK1 and enhancing its kinase activity in complex-I and the necrosome ${ }^{17-21}$. sgRNAs targeting Tnfrsf1a, Mlkl, Ripk3, Cyld, and Spata 2 were not significantly depleted in the time-matched untreated samples in their respective screens, whereas most sgRNAs targeting Ripk1 were weakly depleted, suggesting that RIPK1 may also function independent of TNF $\alpha$ signaling to promote cell proliferation or survival. This RIPK1 pro-survival function may be related to the perinatal lethality of Ripk1-deficient mice caused by induction of apoptosis and necrosis ${ }^{25-27}$. Many of the 112 screen hit genes show even greater levels of sgRNA depletion in the absence of necroptosis induction, including known essential genes such as components of the ribosome (Rpls, Rpl13a) and chaperonin containing TCP1 complex (Cct2), possibly indicating that some mechanisms of generally slowing cell growth can delay
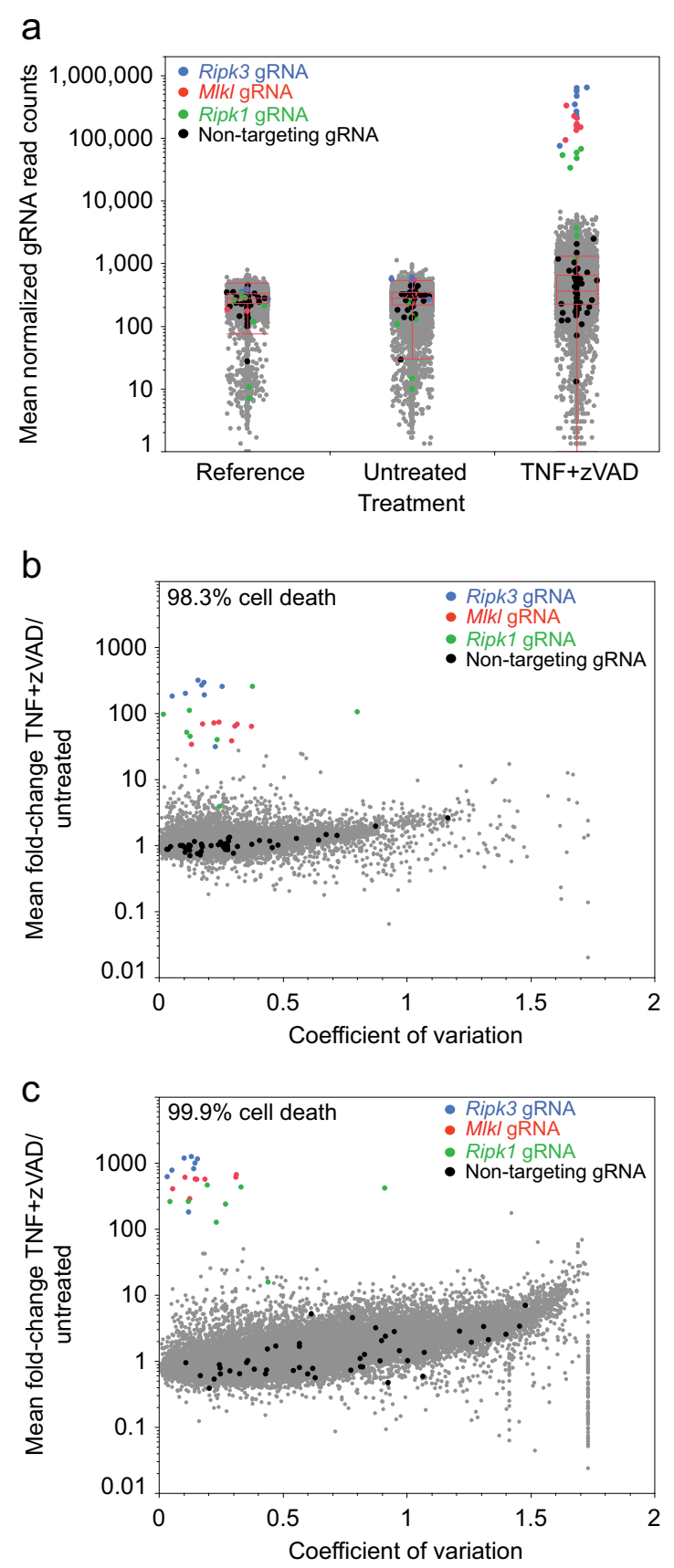

Fig. 1 Positive selection for necroptosis resistance of L929 cells enriches for sgRNAs targeting necrosome component genes. Mean values of all three replicates for each sgRNA in the Library 1 screen are shown, with non-targeting, Ripk1, Ripk3, and Mlkl sgRNAs marked with the indicated colors. a Normalized sgRNA read count distributions for day 2 reference, untreated (day 15), and TNFa+ZVADtreated samples (day 15). b, c Comparison of sgRNA read count mean fold-change and variation between replicate samples for two screens after necroptosis induction producing $98.3 \%$ (b) or $99.9 \%$ (c) cell death

necroptosis. In an attempt to distinguish screen hits more specifically regulating necroptosis, we classified genes based on arbitrary minimum Rho scores for depletion in 


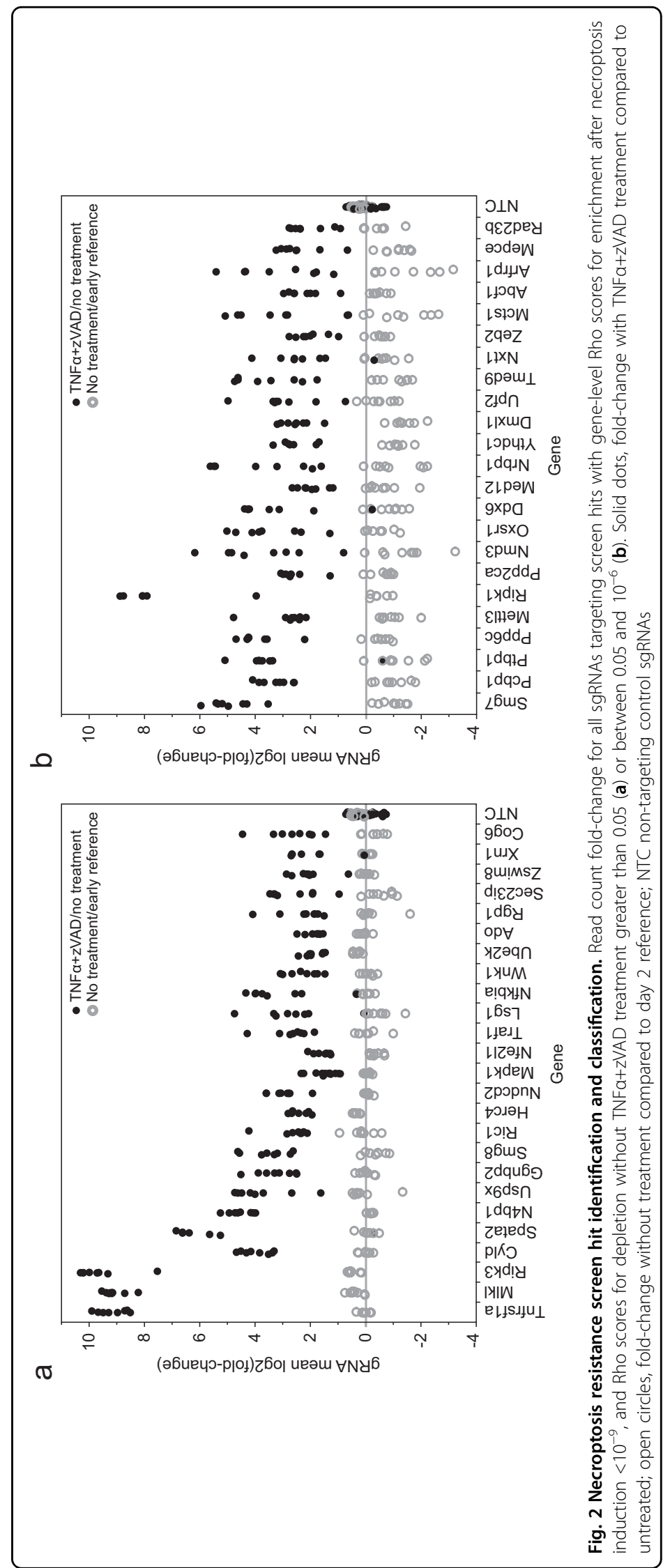




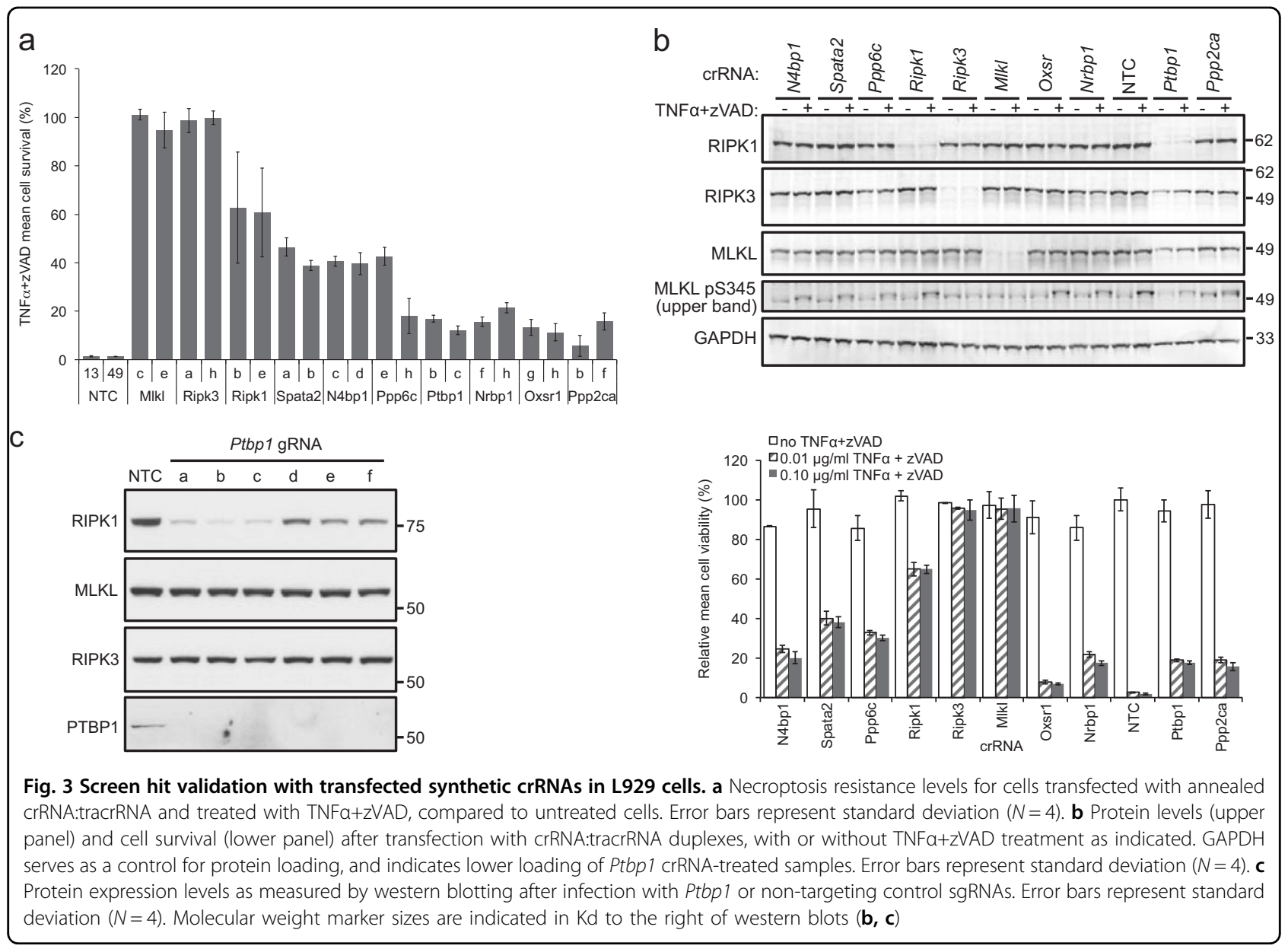

untreated cells (Figure S4b). Twenty-five genes, including Tnfrsf1a, Mlkl, Ripk3, Cyld, and Spata2, have a depletion Rho score greater than 0.05 , suggesting no effect on cell viability or proliferation (Fig. 2a). This group includes N4bp1, which has a level of sgRNA enrichment after necroptosis induction similar to Cyld and Spata2, but has not previously been reported to function in necroptosis. An additional 23 hits have Rho scores between 0.05 and $10^{-6}$ for sgRNA depletion in untreated cells, and this class includes Ripk1 and Ptbp1 (Fig. 2b). A third tier of 17 hits possess Rho scores between $10^{-6}$ and $10^{-8}$ (Table S3).

\section{PTBP1 regulates Ripk1 splicing and expression}

To confirm screen hits, we developed a 96-well format assay for arrayed synthetic crRNAs. crRNAs containing the same targeting sequence as those in the pooled sgRNA libraries were synthesized, annealed with synthetic tracrRNA, and transfected into the L929 Cas9 stable cell line. After 7 days of cell growth to allow for gene knockout, we induced necroptosis with TNF $\alpha$ and zVADfmk before measuring cell viability. crRNAs targeting $M l k l$ and Ripk3 conferred nearly complete resistance to necroptosis, whereas $\sim 60-65 \%$ of cells transfected with
crRNAs targeting Ripk1 were rendered insensitive to the treatment (Fig. 3a, b). Consistent with the screen results, crRNAs against all seven of the novel hit genes tested conferred significant but lower levels of necroptosis resistance, $\sim 25-40 \%$ for Spata2 and N4bp1 (Fig. 3a, b). None of the crRNAs targeting these novel hits reduced cell viability in the absence of necroptosis induction by more than $\sim 15 \%$, although we note that in principle some of these genes might influence cell viability in a manner that is undetectable in a short-term assay (Fig. 3b).

We investigated whether any of these seven new regulators of necroptosis acted by altering expression of necrosome components. crRNA-mediated knockout of Ptbp1 specifically reduced protein levels of RIPK1, but not RIPK3 or MLKL, and this activity is not dependent on stimulation of necroptosis (Fig. 3b, c). crRNAs targeting the other six candidate genes identified in the screen did not clearly affect levels of RIPK1, RIPK3, or MLKL proteins, and did not impair phosphorylation of MLKL at Ser345 upon TNF $\alpha$ and zVAD-fmk stimulation (Fig. 3b). However, we note that the residual RIPK1 protein after Ripk1 or Ptbp1 crRNA transfection can also mediate detectable MLKL phosphorylation, consistent with their 
a

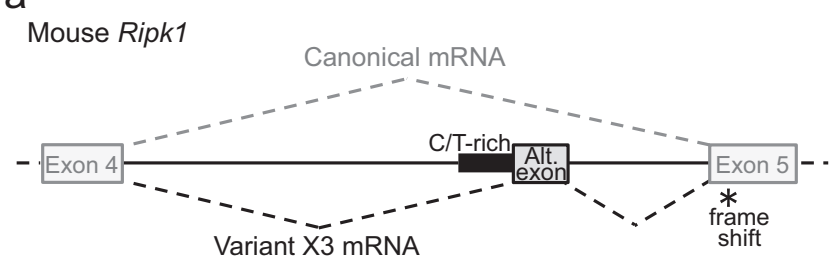

b

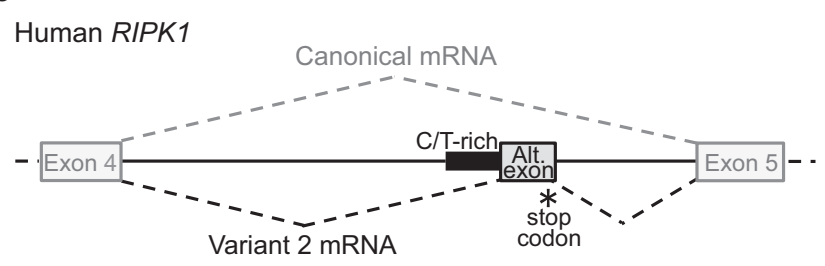

C
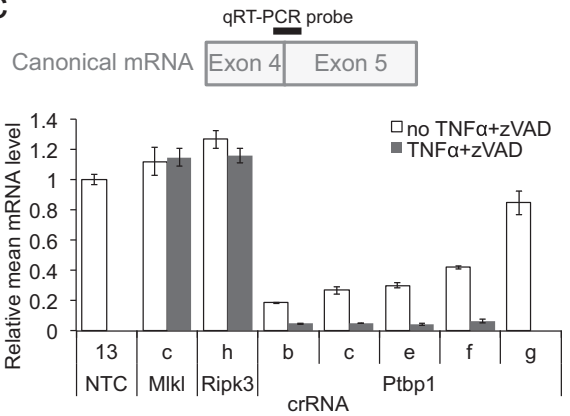

d
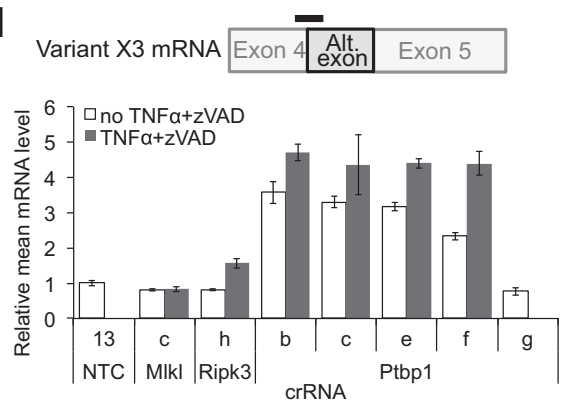

e

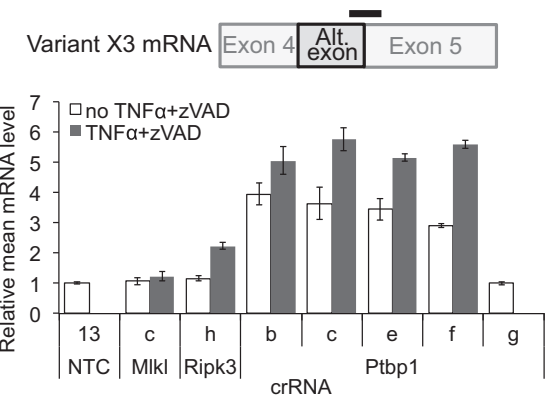

Fig. 4 Ptbp1 regulation of Ripk1 splicing and mRNA expression. a, b Schematic diagram of mouse (a) and human (b) RIPK1 gene splicing between canonical exons 4 and 5, and the alternative exon (Alt. exon). c-e Ripk1 mRNA expression levels, detected by qRT-PCR using primer and probe sets spanning exons as indicated by the bars, after L929 cell transfection of the indicated crRNAs and treated with or without TNFa+zVAD. Expression levels are normalized to Actb mRNA detected in the same sample. Error bars represent standard deviation $(N=2)$. Canonical mRNA, mouse RefSeq Accession NM_009068.3 and human NM_003804.4; Variant X3 mRNA, XM_011244295.2; Variant 2 mRNA, NM_001317061.1; Alt.exon, alternative exon; C/T-rich, putative PTBP1-binding polypyrimidine tracts; ${ }^{*}$, stop codon introduced by alternative splicing

conferring only partial necroptosis resistance, so we cannot definitively conclude that the screen hits act downstream of MLKL activation.

Since PTBP1 is known to control alternative mRNA splicing of certain genes ${ }^{28-30}$, we further explored a potential role for splicing in the mechanism of action of PTBP1 in maintaining RIPK1 protein expression. Multiple Ptbp1 crRNAs that reduce PTBP1 protein levels and confer necroptosis resistance also specifically decrease RIPK1 protein abundance (Fig. 3c). Steady-state levels of Ripk 1 mRNA are reduced by these active Ptbp 1 crRNAs, but not inactive Ptbp1_g crRNA, independent of necrosome activation (Figure S5). The National Center for Biotechnology Information Reference Sequence Database has annotated the mouse Ripk1 transcript variant X3
(RefSeq Accession XM_011244295.2) with an alternative exon between canonical exons 4 and 5 that is spliced to exon 5 to generate a frame shift and premature stop codon (Fig. 4a and S6). Immediately upstream of the $3^{\prime}$ splice site at the alternative exon lies a 139-nt region containing $80 \% \mathrm{C} / \mathrm{U}$ content, characteristic of the polypyrimidine tracts bound by PTBP1 protein to repress cryptic exons $^{29}$ (Figure S6). Using qRT-PCR primers that span the splice junctions of exon 4, exon 5, and the alternative exon, we found that Ptbp1 knockout enhances splicing of exons 4 and 5 to the alternative exon and reduces the more prevalent splicing of exon 4 directly to exon 5 (Fig. 4c-e, S7c, and S7d). qRT-PCR with primers internal to exon 5 (Figure S5b) or exon 11(data not shown) demonstrate that total levels of Ripk 1 mRNAs are 


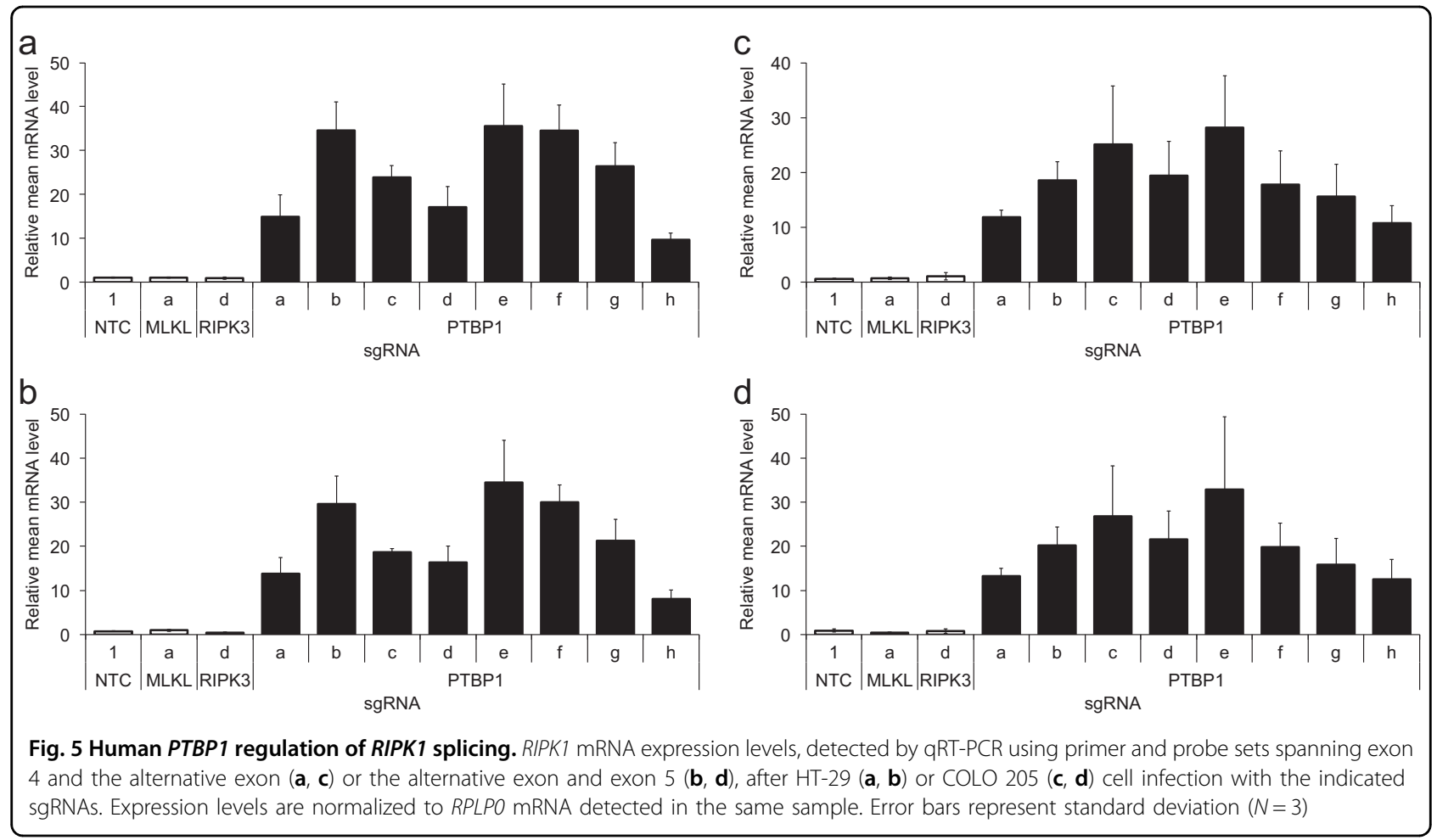

potently diminished by Ptbp 1 crRNAs, suggesting that the enhanced alternative splicing leads to nonsense-mediated decay (NMD) of transcripts that contain the premature termination codon. We conclude that PTBP1 acts as a repressor of a Ripk1 cryptic exon that is deleterious to RIPK1 protein expression.

The cryptic exon and polypyrimidine tract are conserved in human RIPK1, and transcript variant 2 (RefSeq Accession NM_001317061.1) similarly harbors this alternative cassette exon that contains a premature termination codon (Fig. 4a and S6). In two human colorectal adenocarcinoma lines, HT-29 and COLO 205, that are sensitive to necroptosis induced by treatment with TNF $\alpha$, zVAD-fmk, and cIAP inhibitor BV6, multiple sgRNAs targeting PTBP1 enhance inclusion of the RIPK1 alternative exon (Fig. $5 \mathrm{a}-\mathrm{d}$ ). However, the proportion of the alternative splice isoform relative to the canonical splice product that excludes the alternative exon remains $<10 \%$ in these cell lines (Figures S7a and S7b), whereas more than half of the Ripk1 transcripts can contain the alternative exon in mouse L929 cells with Ptbp1 knockout (Figures S7c and S7d). Consequently, we observe minimal or no reduction in RIPK1 canonical splicing of exon 4 to exon 5 (Figure S7e and S7f), or in resistance to necroptosis (Figure S7g and S7h), upon PTBP1 knockout in the human colorectal cancer cell lines.

While necroptosis is not known to function in normal development or tissue homeostasis, necrosome components are expressed in a broad range of tissue types and developmental stages. Our survey of tissue RNA by qRTPCR reveals detectable, though relatively low, levels of the RIPK1 alternative splice product in many human (Figure S8a) and mouse (Figure S8b and S8c) tissues, with higher levels in brain. During embryonic neural development, PTBP1 protein levels decline in neural progenitor cells to initiate an alternative splicing program that mediates neuronal differentiation and PTBP2 expression ${ }^{28-30}$. Comparing mouse cerebral cortex and cerebellar tissues and their precursors, we find that the level of Ripk1 alternative splice isoform is higher in postnatal compared to embryonic stages, when Ptbp1 expression is lower (Figure S8c). To further substantiate this reciprocal relationship, we examined neuronal differentiation of human induced pluripotent stem cells (iPSC). During conversion of the human neural stem cells (NSC) to differentiated neurons, PTBP1 mRNA levels decline to a minimum at day 9 , whereas expression PTBP2 and the RIPK1 alternative splice product increase to a maximum at day 12 (Figure S9a and S9b). Although RIPK1 alternative splicing seems to reflect PTBP1 expression levels, the canonical RIPK1 transcript lacking the alternative exon remains the predominant splice isoform and does not clearly diminish during neuronal differentiation (Figure S9a).

\section{Discussion}

We have executed and rigorously analyzed a positive selection screen of all targetable mouse protein coding sequences for necroptosis resistance using CRISPR/Cas9 gene knockout technology, employing an effective custom 
sgRNA library. The screen identified TNF receptor Tnfrsf1a and known necrosome components Ripk1, Ripk3, and $M l k l$ by strong sgRNA enrichment. More moderate sgRNA enrichment identified RIPK1 deubiquitylase CYLD as a positive regular of necroptosis, consistent with its facilitating RIPK1 translocation from the TNFR1 complex to the necrosome ${ }^{17,22}$, as well as SPATA2 which has subsequently been reported to recruit CYLD to the TNF receptor complex ${ }^{18-21}$. Most importantly, we discovered 20 new genes as necroptosis mediators that show no significant requirement for cell viability in the absence of necroptosis induction, as well as 39 novel necroptosis modulators that are only weakly required for normal cell viability (Table S3). It will be important to determine whether the subpopulation of cells resistant to necroptosis after knockout of these genes can be molecularly defined. Transfection of crRNA: tracrRNA synthetic oligonucleotides confirmed seven novel screen hits that were tested for necroptosis resistance of L929 cells (no other hits were tested).

Previously Hitomi et $\mathrm{al}^{31}$. performed a similar screen for survival of L929 cells treated with zVAD-fmk to induce necroptosis, however this screen utilized siRNAs for gene knockdown and was dependent on autocrine TNF $\alpha$ signaling. Their 432 confirmed screen hits include nine genes that were also identified in our screen: expected genes Tnfrsf1a, Ripk1, Cyld, and Spata2, and unanticipated genes Ptbp1, Pcbp1, Abce1, Atp6ap2, and Zeb2. The Hitomi et $\mathrm{al}^{31}$. screen failed to identify Ripk3 and $M l k l$, suggesting that RNAi off-target activity or incomplete gene depletion may have prevented discovery of other necroptosis mediators. Importantly, treatment with exogenous TNF $\alpha$ in our screen avoids identification of genes required for production of autocrine TNF $\alpha$, whereas the Hitomi et $\mathrm{al}^{31}$. screen identified c-Jun, which is required for TNF $\alpha$ transcription in L929 cells ${ }^{23}$.

Among the extended list of 59 novel screen hits that show moderate to no effect on normal cell viability (Table S3), some are known to function in common biological pathways and processes, suggesting related mechanisms-of-action (Table 1). Like Tnfrsf1a, Ripk1, Cyld, and Spata2, five other screen hits reportedly play various roles in inflammatory and apoptotic signaling. Notably, N4BP1 binds and inhibits the anti-inflammatory and pro-apoptotic ubiquitin E3 ligase ITCH by blocking its interaction with other substrates ${ }^{32}$. Since ITCH can form ubiquitin-editing complexes with the RIPK1 regulators CYLD or $\mathrm{A} 20^{8,33-37}$, there are multiple putative mechanisms for N4BP1 functioning through ITCH to mediate necroptosis. Protein kinase OXSR1 and its direct activator kinase WNK1 were also identified as necroptosis resistance screen hits. This pathway functions in cell osmoregulation to increase water influx and cell volume by both activating $\mathrm{Na}^{+}-\mathrm{K}^{+}-\mathrm{Cl}^{-}$cotransporters and inhibiting $\mathrm{K}^{+}-\mathrm{Cl}^{-}$cotransporters ${ }^{38-40}$. Blocking this pathway may counteract cation influx and cell swelling induced by MLKL and plasma membrane permeabilization to prevent necroptotic cell death.

While retrograde transport of proteins from early endosomes to the trans-Golgi network (TGN) has not previously been implicated in necroptosis, our screen identified eight mediators of this pathway, including both subunits of the RAB6A guanine nucleotide exchange factor (RIC1 and RGP1) ${ }^{41}$ and four subunits of the component of oligomeric golgi complex (COG1, COG3, COG4, and COG6) ${ }^{42}$. Endosome-to-Golgi retrograde transport returns sorting factors from the endo-lysosomal system to the TGN for reuse, thereby maintaining TGN functions of endosome, lysosome, and secretory vesicle biogenesis $^{43,44}$. Two other screen hits, ATP6AP1 and ATP6AP2, are accessory subunits of the vacuolar $\left(\mathrm{H}^{+}\right)$ATPase (V-ATPase) proton pump that mediate acidification of endosomes and lysosomes to maintain their protein sorting and degradation activities ${ }^{45}$. ATP6AP2 (prorenin receptor) also functions as an adaptor between the Wnt receptor and V-ATPase complexes and, along with V-ATPase activity and receptor endocytosis, is required for $W n t / \beta$-catenin signaling ${ }^{46,47}$. TNFR1 complex is internalized into endosomes upon TNF $\alpha$ binding, where CASP8 and other components are recruited and apoptotic signaling is initiated ${ }^{48}$, and can be subsequently routed to the lysosome and TGN, although it is not thought to recycle back to the plasma membrane ${ }^{49}$. We speculate that proper endosome composition and acidification may be required for internalized TNFR1 complex maturation and subsequent necrosome formation.

Another common pathway among five screen hits is nonsense-mediated mRNA decay (NMD) that recognizes and degrades transcripts containing premature termination codons, as well as normal mRNAs with certain other structural features ${ }^{50}$. NMD can selectively target specific mRNAs to suppress the unfolded protein response, oxidative stress response, autophagy, and apoptosis, but has not previously been implicated in regulating necroptosis. Four screen hits are known to regulate mRNA splicing, although through the different transcript features of N6methyladenosine (METTL3 and YTHDC1) ${ }^{51}$, poly(C)rich regions $(\mathrm{PCBP} 1)^{52}$, or polypyrimidine tracts $(\mathrm{PTBP} 1)^{28-30}$. RIPK3 alternative splice products that abolish activity have been identified ${ }^{53}$, although a regulatory role in necroptosis has not been defined.

We demonstrated that the PTBP1 alternative splicing factor specifically maintains expression of RIPK1, and not other necrosome components, by inhibiting inclusion of a cryptic exon that would alter the reading frame, introducing a translation termination codon upstream of the kinase domain. Ptbp1 knockout strongly reduces Ripk1 transcript and protein levels, presumably through mRNA 
Table 1 Potential common mechanisms-of-action identified by new necroptosis regulators

\begin{tabular}{|c|c|c|c|}
\hline Gene class & Gene (alias) & Gene functions ${ }^{a}$ & Protein class or domains ${ }^{a}$ \\
\hline \multirow[t]{5}{*}{$\begin{array}{l}\text { Inflammatory and apoptotic } \\
\text { signaling }\end{array}$} & N4bpl 1 & $\begin{array}{l}\text { Substrate and inhibitor of pro-apoptotic and anti- } \\
\text { inflammatory ITCH, which destabilizes RIPK1 (through } \\
\text { A20) and TAK1 (through CYLD) }\end{array}$ & $\begin{array}{l}\text { KH (RNA binding), UBA (ubiquitin } \\
\text { interaction), and NYN (RNAse) } \\
\text { domains }\end{array}$ \\
\hline & Trafi & $\begin{array}{l}\text { Dimerizes with TRAF2 to mediate TNFR1 activation of } \\
\text { JNK, NF-KB, and anti-apoptotic signals }\end{array}$ & Adaptor protein \\
\hline & Nfkbia (IKBa) & $\begin{array}{l}\text { Blocks nuclear import of NF-KB/Rel, and Bax } \\
\text { recruitment to VDAC1 }\end{array}$ & Ankyrin repeats \\
\hline & Usp9x & Stabilizes ITCH, MCL1, and XIAP & Deubiquitinase \\
\hline & Ube2k (Hip2) & $\begin{array}{l}\text { Promotes TNFa-mediated NF-kB activation; } \\
\text { destabilizes Smac/DIABLO, p53, and Rb }\end{array}$ & Ubiquitin-conjugating enzyme \\
\hline \multirow[t]{2}{*}{ WNK-OSR1/SPAK signaling } & Oxsr1 (Osri) & $\begin{array}{l}\text { Activates } \mathrm{Na}(+)-\mathrm{K}(+)-2 \mathrm{Cl}(-) \text { cotransporters; inhibits } \mathrm{K} \\
(+)-\mathrm{Cl}(-) \text { cotransporters; osmoregulation; ischemic } \\
\text { neuronal cell death }\end{array}$ & Ser/Thr protien kinase \\
\hline & Wnk1 & $\begin{array}{l}\text { Activates OXSR1 and paralog STK39/SPAK; cell volume } \\
\text { recovery during osmotic stress; inhibits autophagy }\end{array}$ & Ser/Thr protein kinase \\
\hline \multirow[t]{11}{*}{$\begin{array}{l}\text { Lysosome-endosome-Golgi-ER } \\
\text { trafficking and function }\end{array}$} & C030046E11Rik (Ric1) & $\begin{array}{l}\text { Retrograde transport from endosomes to Golgi; Ric1- } \\
\text { Rgp1 complex is GEF for late Golgi Rab6A GTPase }\end{array}$ & WD40 repeat \\
\hline & Rgp1 & $\begin{array}{l}\text { Retrograde transport from endosomes to Golgi; Ric1- } \\
\text { Rgp1 complex is GEF for late Golgi Rab6A GTPase }\end{array}$ & \\
\hline & $\operatorname{Cog} 1(L d \mid b)$ & $\begin{array}{l}\text { Intra-Golgi vesicle, endosome-Golgi, and Golgi-ER } \\
\text { retrograde transport to maintain Golgi structure and } \\
\text { function }\end{array}$ & \\
\hline & $\operatorname{Cog} 3(\operatorname{Sec} 34)$ & $\begin{array}{l}\text { Intra-Golgi vesicle, endosome-Golgi, and Golgi-ER } \\
\text { retrograde transport to maintain Golgi structure and } \\
\text { function }\end{array}$ & \\
\hline & $\operatorname{Cog} 4(\operatorname{Cod} 1)$ & $\begin{array}{l}\text { Intra-Golgi vesicle, endosome-Golgi, and Golgi-ER } \\
\text { retrograde transport to maintain Golgi structure and } \\
\text { function }\end{array}$ & \\
\hline & $\operatorname{Cog} 6(\operatorname{Cod} 2, \operatorname{Cdg} 21)$ & $\begin{array}{l}\text { Intra-Golgi vesicle, endosome-Golgi, and Golgi-ER } \\
\text { retrograde transport to maintain Golgi structure and } \\
\text { function }\end{array}$ & \\
\hline & Arfrp1 (Arp1, rl18) & $\begin{array}{l}\text { Retrograde transport from endosomes to Golgi; Golgi- } \\
\text { to-plasma membrane transport }\end{array}$ & Plasma membrane-associated GTPase \\
\hline & Vps29 & $\begin{array}{l}\text { Retrograde transport from endosomes to Golgi or } \\
\text { plasma membrane; retromer complex component }\end{array}$ & $\begin{array}{l}\text { Metallophosphatase superfamily } \\
\text { (inactive) }\end{array}$ \\
\hline & $\begin{array}{l}\text { Tmed9 (Gmp25, } \\
\text { p24alpha2) }\end{array}$ & $\begin{array}{l}\text { Cargo receptor for Golgi-ER retrograde transport } \\
\text { (COPI complex component); promotes ER exit of } \\
\text { proteins }\end{array}$ & \\
\hline & $\operatorname{Sec} 23 i p(p 125 A)$ & $\begin{array}{l}\text { ER-Golgi anterograde transport (COPII complex } \\
\text { component) }\end{array}$ & Phospholipase A1 family; SAM domain \\
\hline & $\begin{array}{l}\text { Atp6ap2 (prorenin } \\
\text { receptor) }\end{array}$ & $\begin{array}{l}\text { Stabilizes V-ATPase; acidification of endodomes and } \\
\text { lysosomes; endolysosomal protein sorting and } \\
\text { degradation; adaptor between V-ATPase and Wnt } \\
\text { receptor complex }\end{array}$ & \\
\hline
\end{tabular}


Table 1 continued

\begin{tabular}{|c|c|c|c|}
\hline Gene class & Gene (alias) & Gene functions ${ }^{a}$ & Protein class or domains ${ }^{a}$ \\
\hline & Atp6ap1 (Ac45) & $\begin{array}{l}\text { Promotes V-ATPases activity; acidification of } \\
\text { endodomes and lysosomes; calcium-dependent } \\
\text { membrane fusion }\end{array}$ & \\
\hline \multirow[t]{5}{*}{$\begin{array}{l}\text { Nonsense-mediated mRNA } \\
\text { decay (NMD) }\end{array}$} & Smg7 & $\begin{array}{l}\text { NMD; stabilizes p53 (promotes ATM phosphorylation } \\
\text { of MDM2) }\end{array}$ & Est1 DNA/RNA binding domain \\
\hline & Smg8 & NMD & \\
\hline & Upf2 & NMD; mRNA nuclear export & \\
\hline & Xrn1 & Decapped mRNA degradation; NMD & Exonuclease \\
\hline & $D d x 6(R c k)$ & $\begin{array}{l}\text { DCP1 mRNA decapping complex activator; mRNA } \\
\text { degradation, translation, and/or translational } \\
\text { repression }\end{array}$ & DEAD-box helicase \\
\hline \multirow[t]{4}{*}{ mRNA splicing } & Ptbp 1 & $\begin{array}{l}\text { Alternative splicing factor; binds mRNA } \\
\text { polypyrimidine tracts }\end{array}$ & RRM (RNA binding) domains \\
\hline & Pcop1 & $\begin{array}{l}\text { mRNA processing; binds mRNA poly }(\mathrm{rC}) \text {; iron } \\
\text { chaperone for metalloproteins }\end{array}$ & $\mathrm{KH}$ (RNA binding) domains \\
\hline & Mett/3 & $\begin{array}{l}\text { N6-methyladenosine }[\mathrm{m}(6) \mathrm{A}]: \mathrm{RNA} \text { methyltransferase; } \\
\text { regulates splicing and mRNA export, translation, and } \\
\text { decay }\end{array}$ & \\
\hline & Ythdc1 (Yt521) & $\begin{array}{l}\text { mRNA methylation }[\mathrm{m}(6) \mathrm{A}] \text { reader protein; alternative } \\
\text { splicing factor }\end{array}$ & YTH [m(6)A-binding] domain \\
\hline
\end{tabular}

${ }^{a}$ Annotations are derived from references cited in the text, or from Gene [Internet] (Bethesda, M): National Library of Medicine, US, National Center for Biotechnology Information; 2017 Jul 09. Available from: https://www.ncbi.nlm.nih.gov/gene/)

NMD. PTBP1 and its paralogs PTBP2 and PTBP3 mediate alternative splicing programs during development of multiple tissues through direct binding to mixed pyrimidine tracts in nascent pre-mRNA to repress splice site recognition, by competing with $\mathrm{U} 2 \mathrm{AF}$ binding to the $3^{\prime}$ splice site as well as additional mechanisms ${ }^{54}$. Most PTBP1-repressed exons are cassette exons that are skipped and contain a CU-rich tract in the intron adjacent to the $3^{\prime}$ splice site ${ }^{29}$, and we have shown this to be the case for Ripk1. A subset of these alternative splicing events also appear to prevent inclusion of a premature stop codon and transcript downregulation by NMD. We have shown that the alternative exon deleterious to RIPK1 protein expression is conserved in the human RIPK1 gene and similarly regulated by PTBP1. PTBP1-dependent Ripk1 alternative splicing does not depend on the induction of necroptosis, and the alternative splice isoform is normally expressed at detectable levels in a variety of human and mouse tissues. Interestingly, inclusion of the RIPK1 deleterious exon increases during differentiation of iPSCderived neural stem cells as PTBP1 levels decline to reprogram the splicing pattern of many genes toward a neuronal cell state. We speculate that this mode of regulating RIPK1 availability might be utilized by some developmental, tissue-specific, or stress response pathways to allow or prevent regulated cell death.

\section{Materials and methods}

\section{Cell culture}

L929 (CCL1-1, ATCC), HT-29 (HTB-38), and COLO 205 (CCL-222) cells were modified to express human codon-optimized Cas9 introduced using the blasticidin selectable pLENTI6.3 Cas9 ${ }^{55}$. L929.pLENTI6.3-Cas9 cells were cultured in Dulbecco's modified Eagle's medium (DMEM) supplemented with $10 \%$ fetal bovine serum (FBS; F6178, Sigma), 2 mM Glutamax (35050061, ThermoFisher), $100 \mathrm{IU}$ penicillin, and $100 \mu \mathrm{g} / \mathrm{ml}$ streptomycin (15140122, Thermofisher), 1\% non-essential amino acids (11140050, ThermoFisher), $10 \mathrm{mM}$ HEPES pH 7.2 and 6 $\mu \mathrm{g} / \mathrm{ml}$ blasticidin (A1113903, ThermoFisher). HT-29. pLENTI6.3-Cas9 and COLO 205.pLENTI6.3-Cas9 cells were cultured in RPMI1640 supplemented with 10\% FBS and $10 \mu \mathrm{g} / \mathrm{ml}$ blasticidin. HEK-293T cells were cultured in DMEM supplemented with 10\% FBS, 2 mM GlutaMAX, and $1 \%$ non-essential amino acids. Human iPSC-derived neural stem cells (MTI-Global Stem, GSC-4311) were 
maintained in NSC maintenance medium (DMEM/F12/ Neurobasal, $1 \times$ GS22, $20 \mathrm{\eta g} / \mathrm{ml}$ bFGF, $20 \mathrm{\eta g} / \mathrm{ml}$ EGF, 20 $\eta \mathrm{g} / \mathrm{ml} \mathrm{BDNF}, 0.11 \mathrm{mM} \beta$-mercaptoethanol, $0.5 \mathrm{mM}$ GlutaMAX) and differentiated in NSC differentiation medium (DMEM/F12/Neurobasal, $1 \times$ GS21, $1 \times \mathrm{N} 2,5 \mu \mathrm{g} / \mathrm{ml}$ cholesterol, $1 \mathrm{mM}$ creatine, $100 \mu \mathrm{M}$ ascorbic acid, $0.5 \mathrm{mM}$ cAMP, $20 \eta \mathrm{g} / \mathrm{ml}$ GDNF, $20 \eta \mathrm{g} / \mathrm{ml}$ BDNF, $1 \mu \mathrm{g} / \mathrm{ml}$ laminin, $0.5 \mathrm{mM}$ GlutaMAX). iPSC neural conversion was monitored by microscopic inspection of cells.

\section{sgRNA sequence design}

RefSeq sequences were downloaded on June 25, 2014, and transcript models identified using GMAP $^{56}$ and Splign $^{57}$ alignments to UCSC mm10. RefSeq CDS annotations were used to determine coding regions. Candidate guide 19-mers with an NGG PAM that were entirely contained in a CDS (including the PAM) and were unique in the genome with respect to both NGG and NAG PAMs were collected. A local version of the MIT algorithm ${ }^{58}$, modified to evaluate only NGG PAMs because of run-time constraints, was used to estimate offtarget scores. Sequences with GC content $<15 \%$ or $>85 \%$, four Ts or three consecutive Ts in the five PAM-proximal positions, four or more consecutive Gs, or homopolymers of five or more bases were eliminated. Eight guides with off-target scores greater than or equal to 70 , hitting the most isoforms for each gene, and with a preference for the $5^{\prime}$ end were selected, resulting in $98.1 \%$ of genes having eight or more guides. For 630 genes tagged as "low-confidence", some restrictions were relaxed in order to obtain at least three guides. Positive controls consisted of 58 genes for which 12 guides were designed and 47 NTC with low similarity to the mouse genome (Table S1).

\section{Pooled CRISPR library design and lentivirus preparation}

In total, 155,120 guides targeting 19,883 mouse genes were optimally selected using a custom sgRNA design algorithm (Table S1; see Extended Methods). The sequences were produced in nine arrayed oligo synthesis pools (Cellecta, Inc.) and sub-cloned into a puromycin selectable lentivirus vector pLKO_SHC201 (Sigma) termed "library vector" in the subsequent text. The combined packaging transfection mix comprised lentivirus library vector, pCMVdR8.9 (expressing gag, pol, and rev genes) and pCMV-VSV-g (expressing envelope protein). Lentivirus libraries were assessed for quality by transducing RKO cells (CRL-2577, ATCC) with serial dilutions of the virus and counting puromycin-resistant cells 5 days post transduction. NGS analysis of sgRNA reference libraries obtained through large-scale transduction of RKO cells confirmed the individual guide proportions to be consistent with the manufacturer's specifications.

\section{Infectious virus titer determination}

In total, $1.11 \times 10^{5}$ cells in a volume of $1 \mathrm{ml}$ were seeded in six-well dishes and incubated overnight at $37^{\circ} \mathrm{C}, 5 \%$ $\mathrm{CO}_{2}$. The virus stock was first diluted $1 / 40$ followed by four five-fold serial dilutions in ice-cold medium containing $48 \mu \mathrm{g} / \mathrm{ml}$ polybrene (TR-1003-G, EMD Millipore) as the virus diluent (for a final concentration of $8 \mu \mathrm{g} / \mathrm{ml}$ ). In total, $0.2 \mathrm{ml}$ of each virus suspension was used to infect five wells, while, a sixth well was treated with $0.2 \mathrm{ml}$ of virus diluent alone. Plates were returned to the incubator for $5 \mathrm{~h}$ incubation after which time each well was replenished to $2 \mathrm{ml}$ with culture medium and incubated a further $48 \mathrm{~h}$. Cells from each virus dilution infection well were harvested, and 10,000 cells in $100 \mu \mathrm{l}$ were re-plated to a 96-well plate. Following overnight incubation, wells were treated with $12.5 \mu \mathrm{l}$ of $72 \mu \mathrm{g} / \mathrm{ml}$ puromycin medium (A1113803, Thermofisher) (for a final concentration of 8 $\mu \mathrm{g} / \mathrm{ml}$ ), and replicate wells were treated with an equivalent volume of medium. After $72-96 \mathrm{~h}$ treatment, the proportion of infected, puromycin-resistant cells was determined by a viability assay using the CellTiter-Glo method, and infectious virus titer was calculated based on the virus volume used (see Supplementary Information Extended Methods).

\section{Screen set-up}

Cells were plated the day before transduction into color-coded vessels to distinguish replicates for processing as three individual experiments that were strictly kept separate for the duration of the cell cultivation process and through NGS analysis (Figure S10). The first of the sub-libraries, which targets the subset of genes within "Library 1", was screened as a proof-of-concept necroptosis resistance positive selection screen. Library gene members and targeting sequences are available in Table S1. L-929.pLENTI6.3-Cas9 cells were infected with a virus dose to achieve a MOI of 0.3 , with sufficient cell numbers plated to obtain a screening depth of 1000 cells per sgRNA, accounting for the estimated proportion of uninfected cells under a Poisson probability distribution. sgRNA representation for the remaining libraries was varied to increase throughput (see Supplementary Information Extended Methods).

\section{sgRNA quantification}

gDNA was isolated using Puregene reagents (158788, Qiagen). DNA concentration was measured using a Qubit assay (Q32853, Molecular Probes). To quantify the abundance of sgRNAs, a gDNA amount consistent with the screening sgRNA representation was used as template for sgRNA amplicon generation by PCR using primer sequences in common with all sgRNAs (see Supplementary Information Extended Methods). NGS read counts of the required depth were obtained and processed using 
gCrisprTools $^{24}$, a modification to previous published methods ${ }^{59}$. sgRNA fold-change relative to the control reference (day 2) or to control expansion (day 15) samples was calculated from size factor-adjusted, median sgRNAscaled raw reads, and then $\log _{2}$ transformed.

\section{Library screen and hit validation micro-scale necroptosis assays}

Necroptosis in L-929.pLENTI6.3-Cas9 cells was induced through media supplementation with murine TNF $\alpha$ (MRRD55, Genentech) to $100 \mathrm{\eta g} / \mathrm{ml}$ combined with Z-VAD-FMK (G7572, Promega) to $20 \mu \mathrm{M}$ (TZ) for 12-16 h, whereas HT-29.pLENTI6.3-Cas9 and COLO 205.pLENTI6.3-Cas9 cells were pre-treated with $200 \mu \mathrm{M}$ Z-VAD-FMK for $2 \mathrm{~h}$ followed by treatment of $10 \mathrm{\eta g} / \mathrm{ml}$ TNF $\alpha$ combined with $1 \mu \mathrm{M}$ BV6 (TZB) for $12-16 \mathrm{~h}$. Necroptosis resistance was assayed in the large-scale screens by sgRNA count enrichment (Table S1), and in the micro-scale assays by the CellTiter-Glo (Promega) viability assay (comparing $\mathrm{TZ}$ or $\mathrm{TZB}$ treated with untreated cultures; see Extended Methods).

\section{crRNA transfection and lentivirus sgRNA transduction}

L-929.pLENTI6.3-Cas9 cells were transfected with crRNA:tracrRNA hybrids at $25 \mathrm{nM}$ in $0.25 \%$ RNAiMax (percent by volume). Successful Cas9-mdiated gene knockout was monitored through visualization of morphology changes for a Cdk1 transfection control. HT-29. pLENTI6.3-Cas9 and COLO 205.pLENTI6.3-Cas9 cells were infected with lentivirus containing sgRNA expression constructs in the presence of $8 \mu \mathrm{g} / \mathrm{ml}$ polybrene at varying MOI. TZ or TZB resistance was monitored to ensure successful control gene knockout. Necroptosis assays were performed at least 5 days after crRNA transfection.

\section{Western blotting}

Cells were processed into lysates in ice-cold Phosphosafe buffer (71296, EMD Millipore) supplemented with dodecyl maltoside to $0.5 \%$ and complete protease inhibitor tabs (11 873580 001, Roche). DNA was sheared by passing the lysates through a 23-G needle. Clarified lysates were normalized for protein content by BCA protein assay (23227, Pierce) prior to denaturation in LDS loading buffer (NP0007, ThermoFisher) supplemented with sample reducing agent (NP0009, Thermofisher), followed by protein separation and transfer to nitrocellulose membranes (see Extended methods). Membranes were blocked in TBS $5 \%$ milk for $2 \mathrm{~h}$ at room temperature. Proteins were immuno-detected by incubation with primary antibodies overnight at $4{ }^{\circ} \mathrm{C}$, followed by compatible speciesspecific secondary infrared fluorescent dye-labeled antiIgG solutions for $2 \mathrm{~h}$ at room temperature, and membrane scanning on an Odyssey imager (Licor) (antibodies are listed in Table S5).

\section{Real-time quantitative PCR assays}

Total RNA was purified using a QIAcube (Qiagen) standard protocol, which includes the DNase digestion step. Mouse Ripk1 transcript was quantified from $25 \eta \mathrm{g} \mathrm{L}-$ 929.pLENTI6.3-Cas9 total RNA, whereas human RIPK1 was quantified from $50 \eta \mathrm{g}$ HT-29.pLENTI6.3-Cas9, COLO 205.pLENTI6.3-Cas9, or iPSC-derived cell total RNA as input to real-time quantitative PCR (RT-qPCR) reactions using Applied Biosystems TaqMan RNA-to-CT reagents (Thermofisher) in a CFX384 Touch Real-Time PCR Detection System (Bio-Rad). Canonically and alternatively spliced Ripk1 RNA transcripts were detected with the gene expression assays listed in Table S6. $\Delta \Delta \mathrm{Ct}$ calculations relative to non-targeting control treatments were used to determine expression levels ${ }^{60}$. Tissue mRNA expression was surveyed using human cDNA arrays (HMRT104 and HBRT101, OriGene) and mouse cDNA arrays (MDRT101 and MNRT101, OriGene) by RT-qPCR according to the manufacturer's instructions.

\section{Acknowledgements \\ We thank Shahrad Daraeikia, Vishva M. Dixit, Brad Friedman, Pete Haverty, Ted Lau, May Liang-Chu, Jessica Lund, Scott Martin, Mark McCleland, Zora Modrusan, Kiran Mukhyala, Yun Qing, Jeremy Stinson, Jianming Wu, Bob Yauch, and Mamie Yu, for scientific discussions, technical assistance, and reagents, as well as Cellecta, Inc., for sgRNA library production.}

\section{Author details}

'Department of Discovery Oncology, Genentech, Inc., 1 DNA Way, South San Francisco, CA 94080, USA. ${ }^{2}$ Department of Bioinformatics and Computational Biology, Genentech, Inc., 1 DNA Way, South San Francisco, CA 94080, USA. ${ }^{3}$ Department of Physiological Chemistry, Genentech, Inc., 1 DNA Way, South San Francisco, CA 94080, USA. ${ }^{4}$ Department of Molecular Biology, Genentech, Inc., 1 DNA Way, South San Francisco, CA 94080, USA. ${ }^{5}$ Department of Biochemical and Cellular Pharmacology, Genentech, Inc., 1 DNA Way, South San Francisco, CA 94080, USA. ${ }^{6}$ Present address: Department of Molecular Biology, Princeton University, Lewis Thomas Laboratory, Washington Road, Princeton, NJ 08544, USA

\section{Conflict of interest}

The authors declare that they have no conflict of interest.

\section{Publisher's note}

Springer Nature remains neutral with regard to jurisdictional claims in published maps and institutional affiliations.

Supplementary Information accompanies this paper at (https://doi.org/ 10.1038/s41419-018-0301-y).

Received: 14 December 2017 Accepted: 4 January 2018 Published online: 15 February 2018

\section{References}

1. Fuchs, Y. \& Steller, H. Programmed cell death in animal development and disease. Cell 147, 742-758 (2011).

2. Linkermann, A., Stockwell, B. R., Krautwald, S. \& Anders, H. J. Regulated cell death and inflammation: an auto-amplification loop causes organ failure. Nat. Rev. Immunol. 14, 759-767 (2014).

3. Linkermann, A. \& Green, D. R. Necroptosis. N. Engl. J. Med. 370, 455-465 (2014). 
4. Brault, M. \& Oberst, A. Controlled detonation: evolution of necroptosis in pathogen defense. Immunol. Cell Biol. 95, 131-136 (2017)

5. He, S., Huang, S. \& Shen, Z. Biomarkers for the detection of necroptosis. Cell. Mol. Life Sci. 73, 2177-2181 (2016).

6. Galluzzi, L., Kepp, O., Chan, F. K. \& Kroemer, G. Necroptosis: mechanisms and relevance to disease. Annu. Rev. Pathol. 12, 103-130 (2017).

7. Tonnus, W. \& Linkermann, A. The in vivo evidence for regulated necrosis. Immunol. Rev. 277, 128-149 (2017).

8. Newton, K. et al. RIPK3 deficiency or catalytically inactive RIPK1 provides greater benefit than MLKL deficiency in mouse mode Is of inflammation and tissue injury. Cell Death Differ. 23, 1565-1576 (2016).

9. Newton, K. RIPK1 and RIPK3: critical regulators of inflammation and cell death. Trends Cell Biol. 25, 347-353 (2015).

10. Grootjans, S., Vanden Berghe, T. \& Vandenabeele, P. Initiation and execution mechanisms of necroptosis: an overview. Cell Death Differ. 24, 1184-1195 (2017).

11. Murphy, J. M. et al. The pseudokinase MLKL mediates necroptosis via a molecular switch mechanism. Immunity 39, 443-453 (2013).

12. Cai, Z. et al. Plasma membrane translocation of trimerized MLKL protein is required for TNF-induced necroptosis. Nat. Cell Biol. 16, 55-65 (2014).

13. Wang, $\mathrm{H}$. et al. Mixed lineage kinase domain-like protein $M L K L$ causes necrotic membrane disruption upon phosphorylation by RIP3. Mol. Cell 54, 133-146 (2014).

14. Silke, J. \& Brink, R. Regulation of TNFRSF and innate immune signalling complexes by TRAFs and CIAPs. Cell Death Differ. 17, 35-45 (2010).

15. Pasparakis, M. \& Vandenabeele, P. Necroptosis and its role in inflammation. Nature 517, 311-320 (2015).

16. Haas, T. L. et al. Recruitment of the linear ubiquitin chain assembly complex stabilizes the TNF-R1 signaling complex and is required for TNF-mediated gene induction. Mol. Cell 36, 831-844 (2009).

17. O'Donnell, M. A. et al. Caspase 8 inhibits programmed necrosis by processing CYLD. Nat. Cell Biol. 13, 1437-1442 (2011).

18. Kupka, S. et al. SPATA2-mediated binding of CYLD to HOIP enables CYLD recruitment to signaling complexes. Cell Rep. 16, 2271-2280 (2016).

19. Wagner, S. A., Satpathy, S., Beli, P. \& Choudhary, C. SPATA2 links CYLD to the TNF-a receptor signaling complex and modulates the receptor signaling outcomes. EMBO J. 35, 1868-1884 (2016).

20. Elliott, P. R. et al. SPATA2 links CYLD to LUBAC, activates CYLD, and controls LUBAC signaling. Mol. Cell 63, 990-1005 (2016).

21. Schlicher, L. et al. SPATA2 promotes CYLD activity and regulates TNF-induced NF-KB signaling and cell death. EMBO Rep. 17, 1485-1497 (2016).

22. Lork, M., Verhelst, K. \& Beyaert, R. CYLD, A20 and OTULIN deubiquitinases in NF-kB signaling and cell death: so similar, yet so different. Cell Death Differ. 24, 1172-1183 (2017).

23. Wu, Y. T. et al. ZVAD-induced necroptosis in L929 cells depends on autocrine production of TNFa mediated by the PKC-MAPKs-AP-1 pathway. Cell Death Differ. 18, 26-37 (2011).

24. Bainer R, Ratman D, Haverty P, Lianoglou S gCrisprTools: Suite of functions for pooled CRISPR screen QC and analysis. R package version 1.4.0. Bioconductor (2017). https://doi.org/10.18129/B9.bioc.gCrisprTools.

25. Rickard, J. A. et al. RIPK1 regulates RIPK3-MLKL-driven systemic inflammation and emergency hematopoiesis. Cell 157, 1175-1188 (2014).

26. Dillon, C. P. et al. RIPK1 blocks early postnatal lethality mediated by caspase-8 and RIPK3. Cell 157, 1189-1202 (2014).

27. Kaiser, W. J. et al. RIP1 suppresses innate immune necrotic as well as apoptotic cell death during mammalian parturition. Proc. Natl Acad. Sci. USA 111, 7753-7758 (2014).

28. Zhang, $X$. et al. Cell-type-specific alternative splicing governs cell fate in the developing cerebral cortex. Cell 166, 1147-1162 (2016).

29. Ling, J. P. et al. PTBP1 and PTBP2 repress nonconserved cryptic exons. Cell Rep. 17, 104-113 (2016).

30. Vuong, J. K. et al. PTBP1 and PTBP2 serve both specific and redundant functions in neuronal pre-mRNA splicing. Cell Rep. 17, 2766-2775 (2016).

31. Hitomi, J. et al. Identification of a molecular signaling network that regulates a cellular necrotic cell death pathway. Cell 135, 1311-1323 (2008).

32. Oberst, A. et al. The Nedd4-binding partner 1 (N4BP1) protein is an inhibitor of the E3 ligase Itch. Proc. Natl Acad. Sci. USA 104, 11280-11285 (2007).

33. Shembade, $\mathrm{N}$. et al. The E3 ligase Itch negatively regulates inflammatory signaling pathways by controlling the function of the ubiquitin-editing enzyme A20. Nat. Immunol. 9, 254-262 (2008).
34. Ahmed, N. et al. The E3 ligase Itch and deubiquitinase Cyld act together to regulate Tak1 and inflammation. Nat. Immunol. 12, 1176-1183 (2011).

35. Wertz, I. E. It takes two to tango: a new couple in the family of ubiquitinediting complexes. Nat. Immunol. 12, 1133-1135 (2011).

36. Venuprasad, K., Zeng, M., Baughan, S. L. \& Massoumi, R. Multifaceted role of the ubiquitin ligase Itch in immune regulation. Immunol. Cell Biol. 93, 452-460 (2015).

37. Draber, $P$. et al. LUBAC-recruited CYLD and $A 20$ regulate gene activation and cell death by exerting opposing effects on linear ubiquitin in signaling complexes. Cell Rep. 13, 2258-2272 (2015).

38. Moriguchi, T. et al. WNK1 regulates phosphorylation of cation-chloridecoupled cotransporters via the STE20-related kinases, SPAK and OSR1. J. Biol. Chem. 280, 42685-44293 (2005).

39. de Los Heros, P. et al. The WNK-regulated SPAK/OSR1 kinases directly phosphorylate and inhibit the $\mathrm{K}+-\mathrm{Cl}-$ co-transporters. Biochem. J. 458, 559-573 (2014).

40. Shekarabi, M. et al. WNK kinase signaling in ion homeostasis and human disease. Cell Metab. 25, 285-299 (2017).

41. Pusapati, G. V., Luchetti, G. \& Pfeffer, S. R. Ric1-Rgp1 complex is a guanine nucleotide exchange factor for the late Golgi Rab6A GTPase and an effector of the medial Golgi Rab33B GTPase. J. Biol. Chem. 287, 42129-42137 (2012).

42. Laufman, O., Hong, W. \& Lev, S. The COG complex interacts directly with Syntaxin 6 and positively regulates endosome-to-TGN retrograde transport. J. Cell Biol. 194, 459-472 (2011).

43. Bonifacino, J. S. \& Rojas, R. Retrograde transport from endosomes to the transGolgi network. Nat. Rev. Mol. Cell Biol. 7, 568-579 (2006).

44. Lu, L. \& Hong, W. From endosomes to the trans-Golgi network. Semin. Cell Dev. Biol. 31, 30-39 (2014).

45. Jansen, E. J. \& Martens, G. J. Novel insights into V-ATPase functioning: distinct roles for its accessory subunits ATP6AP1/Ac45 and ATP6AP2/(pro) renin receptor. Curr. Protein Pept. Sci. 13, 124-133 (2012).

46. Cruciat, C. M. et al. Requirement of prorenin receptor and vacuolar H+-ATPasemediated acidification for Wnt signaling. Science 327, 459-463 (2010).

47. Hagemann, A. I. et al. In vivo analysis of formation and endocytosis of the Wnt/ $\beta$-catenin signaling complex in zebrafish embryos. J. Cell Sci. 127 3970-3982 (2014).

48. Schneider-Brachert, W. et al. Compartmentalization of TNF receptor 1 signaling: internalized TNF receptosomes as death signaling vesicles. Immunity $\mathbf{2 1 ,}$ 415-428 (2004)

49. Watanabe, N. et al. Continuous internalization of tumor necrosis factor receptors in a human myosarcoma cell line. J. Biol. Chem. 263, 10262-10266 (1988).

50. Goetz, A. E. \& Wilkinson, M. Stress and the nonsense-mediated RNA decay pathway. Cell. Mol. Life Sci. 74, 3509-3531 (2017).

51. Wu, R., Jiang, D., Wang, Y. \& Wang, X. N (6)-methyladenosine (m(6)A) methylation in mRNA with a dynamic and reversible epigenetic modification. Mol. Biotechnol. 58, 450-459 (2016).

52. Choi, H. S. et al. Poly(C)-binding proteins as transcriptional regulators of gene expression. Biochem. Biophys. Res. Commun. 380, 431-436 (2009).

53. Yang, Y., Hu, W., Feng, S., Ma, J. \& Wu, M. RIP3 beta and RIP3 gamma, two novel splice variants of receptor-interacting protein 3 (RIP3), downregulate RIP3-induced apoptosis. Biochem. Biophys. Res Commun. 332, 181-187 (2005).

54. Wongpalee, S. P. et al. Large-scale remodeling of a repressed exon ribonucleoprotein to an exon definition complex active for splicing. Elife 5, e19743 (2016).

55. Tan, J. \& Martin, S. E. Validation of synthetic CRISPR reagents as a tool for arrayed functional genomic screening. PLOS ONE 11, e0168968 (2016).

56. Wu, T. D. \& Watanabe, C. K. GMAP: a genomic mapping and alignment program for mRNA and EST sequences. Bioinformatics 21, 1859-1875 (2005).

57. Kapustin, Y., Souvorov, A., Tatusova, T. \& Lipman, D. Splign: algorithms for computing spliced alignments with identification of paralogs. Biol. Direct 3, 20 (2008).

58. Hsu, P. D. et al. DNA targeting specificity of RNA-guided Cas9 nucleases. Nat. Biotechnol. 31, 827-832 (2013).

59. Li, W. et al. MAGeCK enables robust identification of essential genes from genome-scale CRISPR/Cas9 knockout screens. Genome Biol. 15, 554 (2014).

60. Livak, K. J. \& Schmittgen, T. D. Analysis of relative gene expression data using real-time quantitative PCR and the 2- $\triangle C T$ method. Methods 25, 402-408 (2001). 